\title{
ARTÍCULOS
}

\section{DISEÑO DE INFORMACIÓN DIGITAL: REVISIÓN Y CLASIFICACIÓN DE INDICADORES HEURISTICOS PARA CONTENIDOS WEB}

\section{Digital information design: Web content heuristics review and classification}

Lucía Jiménez-Iglesias, Mario Pérez-Montoro y Lydia Sánchez-Gómez
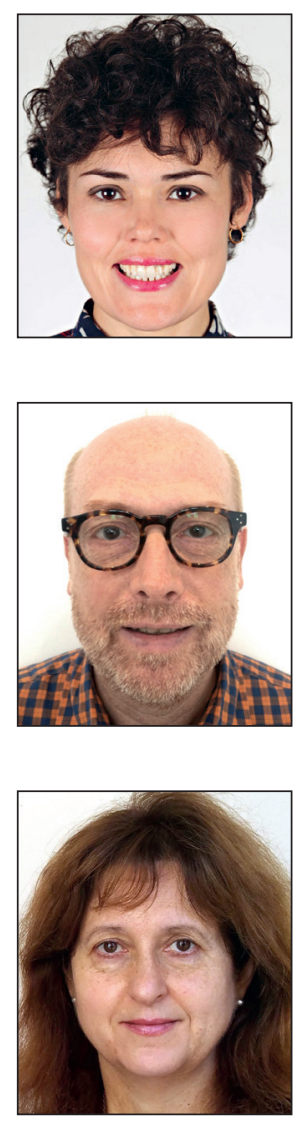

Lucía Jiménez-Iglesias es doctoranda en el Departamento de Biblioteconomía, Documentación y Comunicación Audiovisual de la Universitat de Barcelona (UB), donde se incorporó en 2015 como investigadora en formación tras realizar el máster en Sociedad de la Información y el Conocimiento (UOC). Ejerce como profesora ayudante en el grado de comunicación audiovisual de la UB, donde es parte del grupo Dhigecs. Sus líneas de investigación incluyen el diseño de información digital y las nuevas plataformas mediáticas.

http://orcid.org/0000-0001-8831-8600

luciajimenez@ub.edu

Mario Pérez-Montoro es doctor en filosofía y ciencias de la educación por la Universitat de Barcelona y posgraduado en organización de sistemas de documentación por la Universidad Politécnica de Catalunya. Ha realizado estudios de posgrado en el Instituto di Discipline della Comunicazione (Università di Bologna), y ha sido profesor visitante del CSLI (Stanford University, California) y de la School of Information (UC Berkeley, California). Es profesor del Departamento de Biblioteconomía, Documentación y Comunicación Audiovisual de la Universidad de Barcelona. http://orcid.org/0000-0003-2426-8119

perez-montoro@ub.edu

Lydia Sánchez-Gómez es profesora agregada en Comunicación Audiovisual en la Facultad de Biblioteconomía y Documentación de la Universitat de Barcelona. Doctora en filosofía por la Stanford University, centra su investigación en la calidad democrática de los medios de comunicación, la epistemología y la teoría de la comunicación.

http://orcid.org/0000-0001-7814-0087

Isanchezg@ub.edu

Universitat de Barcelona Departamento de Biblioteconomía, Documentación y Comunicación Audiovisual Melcior de Palau, 140. 08014 Barcelona, España

\section{Resumen}

Desde la aparición de Internet se ha producido una continua evolución en las interfaces, intentándose ofrecer una buena experiencia a los usuarios. Los profesionales implicados en el diseño y desarrollo de sitios web recurren en muchos casos a los indicadores heurísticos que se han ido recopilando durante años de investigación y praxis. Sin embargo, tales indicadores presentan características heterogéneas, siendo complicado localizar los que son aplicables en cada caso. El objetivo de esta investigación es identificar y analizar, mediante una revisión de mapeo, los indicadores heurísticos con mayor relevancia científica y geográfica, y clasificarlos según diferentes elementos formales y de contenido. El resultado son dos taxonomías que facilitan la localización, análisis y aplicación de los indicadores, y que constituyen un instrumento para que los profesionales e investigadores del ámbito aborden la evaluación de interfaces web.

\section{Palabras clave}

Diseño de información; Diseño web; Sitios web; Contenidos; Experiencia de usuario; Diseño centrado en el usuario; Diseño de interacción; Usabilidad; Indicadores heurísticos; Directrices; Revisión; Taxonomías; Revisión bibliográfica. 


\begin{abstract}
Since the emergence of the Internet there has been continuous evolution in interface design, with designers trying to offer a good experience to users. Website developers regularly turn to heuristic indicators that have been collected throughout years of research and praxis. Nevertheless, these indicators are diverse and it is complicated locating which ones are applicable in every case. The objective of this work is to identify and analyze the most scientifically and geographically relevant heuristic indicators through a mapping review, in order to classify them according to formal and content components. The results are two taxonomies which make it easier to identify the location, and analyze and apply the guidelines.
\end{abstract}

\title{
Keywords
}

Information design; Web design; Web sites; Content; User experience; UX; User-centered design; Interaction design; Usability; Heuristics; Guidelines; Review; Taxonomies; Bibliographic review.

Jiménez-Iglesias, Lucía; Pérez-Montoro, Mario; Sánchez-Gómez, Lydia (2017). “Diseño de información digital: revisión y clasificación de indicadores heurísticos para contenidos web". El profesional de la información, v. 26, n. 6, pp. 10291046.

https://doi.org/10.3145/epi.2017.nov.03

\section{Introducción}

Desde la aparición de la World Wide Web en 1990, el uso de internet no ha parado de aumentar. Millones de personas en todo el mundo utilizan esta Red con propósitos muy distintos: para comunicarse, para acceder a información de actualidad o para hacer compras online. En los últimos años se han diversificado los dispositivos de acceso y los smartphones se han convertido en el método más habitual para navegar por la red: según la AIMC (2017) los utiliza un 95,4\% de internautas.

Las interfaces web han evolucionado, buscando ofrecer una navegación cómoda y eficiente. Para que un sitio web tenga éxito es necesario que ofrezca una buena experiencia a los usuarios, y que estos logren su objetivo teniendo una experiencia global positiva. Por eso optimizar la experiencia de usuario (user experience, UX) es el objetivo de todas las disciplinas implicadas en el diseño y desarrollo de una web (el diseño centrado en el usuario-DCU-, el diseño de interacción o la usabilidad, entre otras).

Este proceso requiere una coordinación eficaz de todos los profesionales involucrados en la creación del sitio. Para conseguirlo, estos profesionales recurren en muchos casos a indicadores recopilados durante años de investigación y praxis, y que se utilizan para ejecutar el método de inspección de la usabilidad conocido como evaluación heurística (Nielsen; Molich, 1990) o análisis de expertos. En este método se utilizan reglas establecidas para analizar la calidad de una web (Pedraza-Jiménez; Codina; Guallar, 2016), permitiendo implementar mejoras tras una valoración rápida y poco costosa (Donker-Kuijer; De-Jong; Lentz, 2010).

Existen numerosas formas de referirse a los tipos de publicaciones cuyo objetivo es guiar el análisis y evaluación de un sitio web: indicadores heurísticos, protocolos, principios, directrices... Según Mariage, Vanderdonckt y Pribeanu (2005), las primeras directrices aparecieron en la década de los ochenta, cuando el uso de ordenadores comenzaba a expandirse, como los trabajos de Smith y Mosier (1986), Shneiderman y Plaisant (1987) o Norman (1988). En los años noventa, la publicación de este tipo de protocolos ex- perimentó un crecimiento notable debido a la expansión de la propia internet. Mariage, Vanderdonckt y Pribeanu (2005) apuntan que comenzaron a elaborarse directrices dirigidas a interfaces web, bien transformando algunos de los indicadores existentes para adaptarlos al nuevo entorno o introduciendo nuevos temas: Nielsen y Molich (1990, posteriormente ampliado en Nielsen, 1994), Mayhew (1992), Constantine (1995) o Brown (1998). Desde entonces, y dado el constante desarrollo de la tecnología web, han seguido apareciendo publicaciones con el objetivo de evaluar el diseño de interacción, las interfaces de usuario, el DCU y la usabilidad, como Nielsen y Tahir (2002), Tognazzini (2003), González, Lorés y Granollers (2008), Väänänen-Vainio-Mattila y Wäljas (2009), Suárez-Torrente et al. (2013), así como investigaciones basadas en interfaces móviles, como Yáñez-Gómez, Cascado-Caballero y Sevillano (2014), Inostroza et al. (2016) o García-López et al. (2017).

\section{El término UX abarca todo el proceso de interacción de un usuario con un sistema}

Existe por tanto una gran diversidad de indicadores heurísticos con distinto origen, formato, finalidad y contenido, de manera que en muchas ocasiones resulta complicado localizarlos y saber cuáles son aplicables en cada caso.

El objetivo de este trabajo es proporcionar una taxonomía de las directrices más relevantes para facilitar a investigadores y profesionales su localización, análisis y aplicación. Además de constituir un instrumento de trabajo con el que afrontar la evaluación de una web, la taxonomía aporta diferentes niveles (formales y de contenido) de análisis, contribuyendo así a estructurar este ámbito de estudio.

\section{Marco teórico}

Para comprender la utilidad y aplicación de los indicadores heurísticos analizados, es necesario desgranar previamente el significado de UX y de otras disciplinas afines, como son el diseño centrado en el usuario (DCU), el diseño de interacción y la usabilidad. 


\subsection{UX, experiencia de usuario}

El término UX abarca todo el proceso de interacción de un usuario con un sistema. Incluye

"todas las emociones, creencias, preferencias, percepciones, respuestas físicas y psicológicas, comportamientos y logros del usuario que ocurren antes, durante y tras el uso" de ese sistema (ISO, 2010, p. 3).

UX no sólo comprende las expectativas, necesidades y motivaciones del usuario, sino también las características del sistema y el contexto en que es usado (Pereira-Da-Silva; De-Souza; Maciel, 2016). Algunos autores afirman además que una buena UX no se construye sólo desde el punto de vista del usuario, sino que también ha de satisfacer las necesidades de la organización creadora del producto o servicio (Kuniavsky, 2010).

\section{Ofrecer una buena experiencia global} a los usuarios constituye el objetivo de toda web

Ofrecer una buena experiencia global a los usuarios constituye el objetivo de toda web. Por eso la finalidad última de las evaluaciones de expertos es valorar si la web analizada lo logra. Para materializarse, la UX necesita la correcta implementación y coordinación de otras disciplinas: el DCU por un lado, y el diseño de interacción y la usabilidad por otro.

EI DCU supone una aproximación holística al diseño de una web, de manera que durante todo el proceso (desarrollo, puesta a disposición del público y evaluación) se tiene en cuenta al usuario final: sus características, necesidades, limitaciones o el contexto en el que utilizará el diseño (Garreta-Domingo; Mor-Pera, 2010). Se trata de una filosofía de diseño que sitúa en el centro del proceso de diseño a las personas que utilizarán el sistema (ISO, 2010, p. 14), con el objetivo de garantizarles una experiencia global eficiente y satisfactoria (Garrett, 2010).

El diseño de interacción analiza el comportamiento de las personas y de los sistemas durante su interacción, tratando de optimizar esa re-

lación interactiva en la fase de desarrollo (Hassan-Montero, 2013).

La usabilidad se refiere a la facilidad de uso de un sistema y mide hasta qué punto los usuarios lo utilizan con eficacia, eficiencia y satisfacción (ISO, 2010).

DCU, diseño de interacción y usabilidad se centraban originalmente en el desarrollo de los aspectos más formales de los sistemas. No obstante, en la década de los
2000 debido a la difusión de internet y de los nuevos dispositivos de acceso, las tres disciplinas comenzaron también a volver su mirada hacia la UX e incorporaron a su análisis elementos emocionales y contextuales más complejos para poder abarcar así la experiencia total del usuario (Wright; Blythe, 2007).

Dado que en la bibliografía no están claras las diferencias entre estas disciplinas, la revisión realizada ha planteado la necesidad de definir con precisión el ámbito de aplicación de cada una de ellas. Para ello se propone discernir en qué fases del proceso de interacción entre un usuario y una web interviene cada disciplina, como se puede observar en la figura 1.

Siguiendo la definición de UX, el proceso de interacción de un usuario con una web comprende todas las percepciones que experimenta antes, durante y después de utilizarla (ISO, 2010). En este proceso se pueden diferenciar dos áreas:

- el desarrollo de la web, y

- la experiencia del usuario en sí.

DCU, diseño de interacción y usabilidad están asociados al desarrollo de la web; el DCU se extiende durante las tres fases, mientras que el diseño de interacción sólo interviene durante las fases de inicio e interacción y la usabilidad únicamente durante la fase de interacción del usuario con la web. La UX forma parte del entorno del usuario y se extiende durante todo el proceso, trascendiendo el enfoque del DCU e incluyendo aspectos que van más allá de la funcionalidad (Väänänen-Vainio-Mattila; Wäljas, 2009).

\subsection{Indicadores heurísticos sobre diseño y desarrollo web}

El objetivo de los indicadores heurísticos es guiar a desarrolladores, expertos e investigadores en la evaluación de interfaces web (Bevan; Spinhof, 2007). Estos protocolos conforman un método aceptado con el que recopilar el conocimiento adquirido y darle forma de buenas prácticas para guiar la evaluación de futuros diseños (Henninger, 2000). Bevan y Spinhof (2007) señalan que un buen conjunto de directrices debe incluir indicadores generales sobre aspectos genéricos de la interfaz y heurísticos más específicos para realizar una evaluación directa.

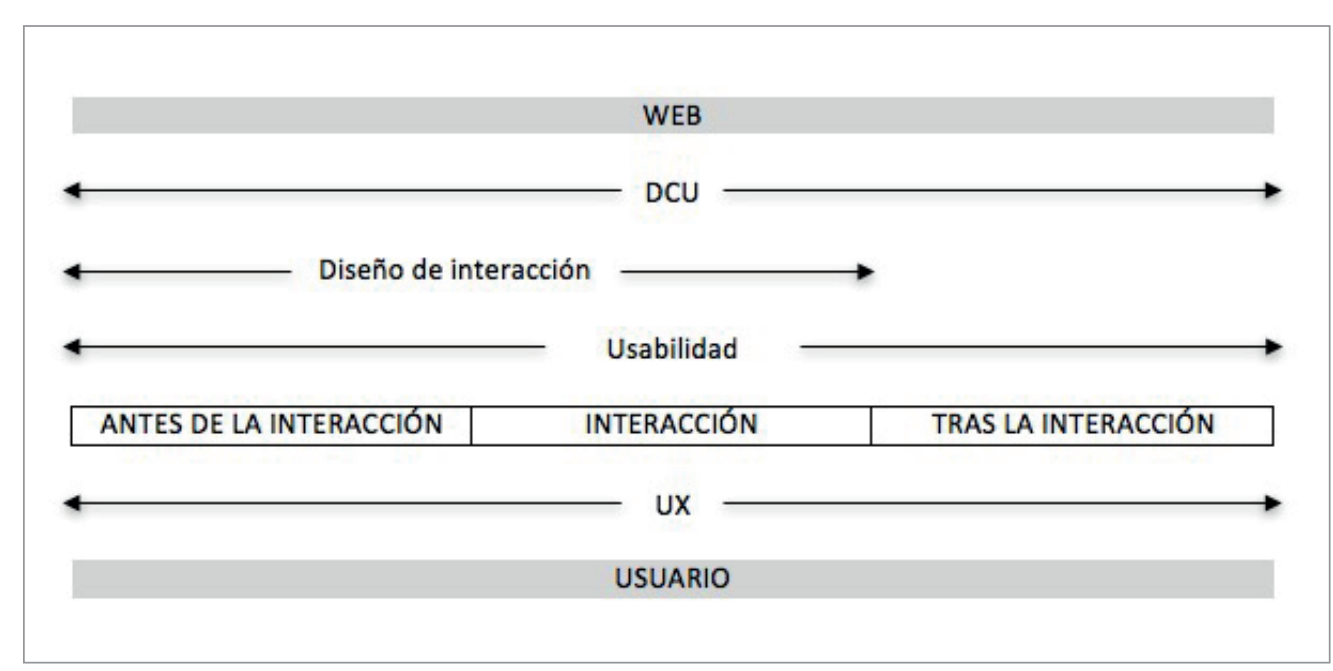

Figura 1. Disciplinas involucradas en el proceso de interacción usuario-web. 
Existen diferentes tipos de indicadores heurísticos en función de las propuestas que integran, que pueden ser principios, directrices o convenciones (Ohnemus, 1997; Mariage; Vanderdonckt; Pribeanu, 2005):

- principios son estipulaciones generales que guían las decisiones de diseño durante la fase de desarrollo;

- directrices son enunciados más concretos, basados en los principios, pero aplicables a un ámbito concreto de diseño;

- convenciones son recomendaciones específicas dirigidas a resolver un problema concreto.

Aunque los indicadores heurísticos son útiles para la evaluación de una web, su aplicación no está exenta de problemas

(Bevan, 2005). Bevan y Spinhof (2007) consideran que

"la mayoría de los estándares actuales todavía no son totalmente útiles en las revisiones de directrices" (p. 9),

y Al-Badi, Ali y Al-Balushi (2012) señalan entre sus limitaciones el origen heterogéneo del lenguaje con el que están redactados y la dificultad para saber cuándo y cómo aplicarlos. Ante este problema, Henninger (2000) propone un sistema para localizar las directrices específicas que pueden tenerse en cuenta en la evaluación de una interfaz concreta. Mariage, Vanderdonckt y Pribeanu (2005) también afirman que es complicado localizar los protocolos aplicables a un contexto web específico, dada la gran variedad de directrices y las diferencias entre ellas. Estos autores señalan además que no existe ninguna guía para ayudar a los profesionales a localizar y recopilar las directrices aplicables en cada caso.

\section{La presente investigación es una revisión} actualizada de los indicadores heurísticos para la evaluación de interfaces web

Con el objetivo de facilitar el trabajo de profesionales e investigadores, se han realizado algunas revisiones de la bibliografía para otorgar orden a estas directrices. Destaca el trabajo de Ohnemus (1997), que recopiló un conjunto de guías de estilo relevantes del momento y las clasificó en función de su origen (guías de estilo procedentes de webs, otro tipo de directrices presentes en Internet y libros de diseño).

La presente investigación es una revisión actualizada de los indicadores heurísticos para la evaluación de interfaces web. El objetivo es recopilar los protocolos más relevantes y elaborar una clasificación que sirva como referencia para los profesionales e investigadores interesados en estas publicaciones. El resultado puede ayudar a los profesionales a localizar los heurísticos necesarios para la evaluación de sitios web, aportando una categorización útil para las futuras aproximaciones académicas a este tema.

\section{Objetivos y metodología}

La finalidad de este trabajo es proporcionar una taxonomía de los indicadores heurísticos más relevantes que facilite a los investigadores y profesionales su localización, análisis y aplicación.

Para lograrlo, se plantean los siguientes objetivos específicos:

- Identificar los principales indicadores heurísticos para la evaluación de interfaces web por parte de expertos.
- Analizar cada uno de los documentos y clasificarlos en función de aspectos formales (autor, fecha, país y tipo de publicación) y de contenido (estructura, orientación a dispositivos móviles, evidencia empírica, existencia de una checklist de directrices y sistemas de arquitectura de la información).

- Elaborar una taxonomía de las directrices que facilite a los investigadores y profesionales su identificación, análisis y aplicación.

El punto de partida para elaborar la taxonomía es una revisión de mapeo o mapeo sistemático, un método que permite explorar y categorizar las publicaciones de un área para obtener una visión de conjunto, de manera que es posible realizar una descripción amplia y profunda de la bibliografía, e identificar los temas que aún no han sido abordados para llevar a cabo futuras investigaciones (Grant; Booth, 2009).

En este caso, el mapeo sistemático de la bibliografía se ha llevado a cabo a lo largo de tres fases que se corresponden con los tres objetivos específicos planteados en esta investigación: las dos primeras (identificación y análisis) se explican en los apartados 3.1 y 3.2, y la tercera (elaboración de una taxonomía) está incluida en el apartado 4 (resultados) de este trabajo.

\subsection{Identificación de los indicadores heurísticos}

El primer paso para abordar la revisión de mapeo consiste en identificar los indicadores heurísticos para la evaluación de interfaces web que han tenido una relevancia significativa en los últimos años y que están ampliamente aceptados. El criterio fundamental de selección es la repercusión profesional, geográfica y científica.

Las directrices se pueden clasificar en cinco categorías según su origen:

- Institucionales: elaboradas por organizaciones internacionales.

- Gubernamentales: elaboradas por entidades gubernamentales.

- De expertos: elaboradas por expertos.

- Online: elaboradas por expertos o asociaciones de expertos y publicadas en sus webs.

- Científicas: procedentes de la bibliografía científica.

Las directrices institucionales analizadas en este trabajo proceden de dos organizaciones internacionales de prestigio: el World Wide Web Consortium (W3C) y la Organización Internacional de Normalización (ISO), que han publicado directrices para optimizar la experiencia de usuario en entornos web.

Tabla 1. Directrices institucionales

\begin{tabular}{|l|l|}
\hline Organización & \multicolumn{1}{c|}{ Directriz } \\
\hline W3C & $\begin{array}{l}\text { Mobile web best practices 1.0 (Rabin; McCathieNe- } \\
\text { vile, 2008) }\end{array}$ \\
\hline W3C & $\begin{array}{l}\text { Mobile web application best practices (Connors; } \\
\text { Sullivan, 2010) }\end{array}$ \\
\hline ISO & $\begin{array}{l}\text { ISO 9241-151:2008. Directrices para las interfaces de } \\
\text { usuario web (ISO, 2008) }\end{array}$ \\
\hline ISO & $\begin{array}{l}\text { ISO 9241-210:2010. Diseño centrado en el operador } \\
\text { humano para los sistemas interactivos (ISO, 2010) }\end{array}$ \\
\hline
\end{tabular}


Tabla 2. Directrices gubernamentales

\begin{tabular}{|l|l|}
\hline \multicolumn{1}{|c|}{ País } & \multicolumn{1}{c|}{ Directriz } \\
\hline Unión Europea & Information providers guide (European Commission, s.f.) \\
\hline Reino Unido & Illustrated handbook for web management teams (Office of the e-Envoy Cabinet Office, 2003) \\
\hline EUA & Research-based web design \& usability guidelines (HSS; GSA, 2006) \\
\hline Suecia & Swedish national guidelines for public sector websites (Verva, 2006) \\
\hline India & Guidelines for Indian government websites (Government of India, 2009) \\
\hline Tasmania & Web usability guidelines (Office of eGovernment, 2010b) / Web design and navigation guidelines (Office of eGovernment, 2010a) \\
\hline Colombia & Directrices de usabilidad para sitios web del Estado colombiano (Carvajal; Saab, 2010) \\
\hline Chile & Guía web (Gobierno de Chile, 2012) \\
\hline Nueva Zelanda & Web usability standard 1.2 (New Zealand Government, s.f.) \\
\hline EUA & US web design standards (CIO, 2015) \\
\hline Australia & Digital service standard. Digital Transformation Office (2015) \\
\hline Reino Unido & Service manual (Gob.UK, 2015) \\
\hline
\end{tabular}

Tabla 3. Directrices de expertos

\begin{tabular}{|l|l|l|}
\hline \multicolumn{1}{|c|}{ Autor(es) } & \multicolumn{1}{c|}{ Año } \\
\hline Norman & Seven principles for transforming difficult tasks into simple ones (user-centered design) & \multicolumn{1}{|c|}{1988} \\
\hline Mayhew & Principios de Deborah Mayhew \\
\hline Nielsen & 10 usability heuristics for user interface design \\
\hline Constantine & What do users want? Engineering usability into software \\
\hline Brown & Human-computer interface design guidelines \\
\hline Hassan-Montero; Martín-Fernández & Guía de evaluación heurística de sitios web \\
\hline Tognazzini & First principles of interaction design (revised \& expanded) \\
\hline Krug & Don't make me think (revisited). A common sense approach to web and mobile usability & \multicolumn{2}{|c|}{2014} \\
\hline Rosenfeld; Morville; Arango & Information architecture for the web and beyond \\
\hline Shneiderman et al. & The eight golden rules of interface design \\
\hline Pedraza-Jiménez; Codina; Guallar & Sistema articulado de análisis de medios digitales (Saamd) \\
\hline
\end{tabular}

Tabla 4. Directrices online

\begin{tabular}{|l|l|c|}
\hline \multicolumn{1}{|c|}{ Autor(es) } & \multicolumn{1}{c|}{ Directriz } \\
\hline Google; Answer Lab & Principles of mobile site design & s.f. \\
\hline Van-Welie & Patterns in interaction design (Pattern library) & 2008 \\
\hline UXPA & Principles for usable design & 2011 \\
\hline Toxboe & User interface design pattern library & 2011 \\
\hline
\end{tabular}

Dada su autoridad y relevancia geográfica, se han recopilado también los indicadores heurísticos publicados por entidades gubernamentales de todo el mundo (tabla 2). Este tipo de directrices tiene por objetivo guiar el desarrollo de las webs oficiales de un país para mejorar los servicios que el gobierno ofrece a los ciudadanos a través de las nuevas tecnologías (Donker-Kuijer; De-Jong; Lentz, 2010). Los principios que recogen se han incluido en este trabajo porque las directrices que plantean pueden extrapolarse a otros ámbitos.

También se han considerado los principios elaborados por expertos en experiencia de usuario, DCU, diseño de interacción y usabilidad que han tenido una influencia conside- rable en este campo durante los últimos años (tabla 3). Es importante tener en cuenta estas directrices dada su popularidad y repercusión.

Respecto a las directrices online, se trata de principios procedentes de webs que han sido publicados por expertos y/o asociaciones de expertos en sus webs o blogs (tabla 4).

Por último, se realizó una revisión de las publicaciones científicas recientes para localizar los trabajos con mayor impacto (tabla 5). En este caso, la planificación de la búsqueda se basó en el framework FDC (facetar, derivar, combinar) propuesto por Codina (2016) y la exploración se realizó en las principales bases de datos científicas (Web of Science y Scopus). 
Tabla 5. Directrices científicas

\begin{tabular}{|l|l|l|}
\hline \multicolumn{1}{|c|}{ Autor(es) } & \multicolumn{1}{c|}{ Directriz } & Año \\
\hline Sutcliffe & Assessing the reliability of heuristic evaluation for website attractiveness and usability & 2002 \\
\hline Badashian et al. & Fundamental usability guidelines for user interface design & 2008 \\
\hline Väänänen-Vainio-Mattila; Wäljas & Development of evaluation heuristics for web service user experience & 2009 \\
\hline Lobo et al. & Web usability guidelines for smartphones: A synergic approach & 2011 \\
\hline Suárez-Torrente et al. & Sirius: A heuristic-based framework for measuring web usability adapted to the type of website & 2013 \\
\hline $\begin{array}{l}\text { Yáñez-Gómez; Cascado-Caballe- } \\
\text { ro; Sevillano }\end{array}$ & Heuristic evaluation on mobile interfaces: a new checklist & 2014 \\
\hline $\begin{array}{l}\text { Chuan; Sivaji; Ahmad } \\
\text { De-Almeida-Pacheco; De-Almei- } \\
\text { da-Souza-Concilio }\end{array}$ & Proposed usability heuristics for testing gestural interaction & 2014 \\
\hline Joyce; Lilley & Externalist philosophy and interaction: Proposal of heuristics for the design of interactive systems & 2014 \\
\hline Inostroza et al. & Towards the development of usability heuristics for native smartphone mobile applications & 2014 \\
\hline $\begin{array}{l}\text { Pereira-Da Silva; De Souza; } \\
\text { Maciel }\end{array}$ & Developing Smash: A set of SMArtphone's uSability Heuristics & 2016 \\
\hline García-López et al. & Establishing guidelines for user quality of experience in ubiquitous systems & 2016 \\
\hline & Validation of navigation guidelines for improving usability in the mobile web & 2017 \\
\hline
\end{tabular}

\subsection{Caracterización}

Una vez identificadas las directrices, el siguiente paso consistió en extraer de cada una de ellas dos niveles de información, ampliando así la revisión de Donker-Kuijer; De-Jong y Lentz (2010): elementos formales y elementos de contenido.

La primera dimensión proporciona información formal básica sobre cada una de las publicaciones y permite dibujar una panorámica de las directrices existentes. Por su parte, el nivel de contenido constituye un acercamiento cualitativo a las directrices cuyo objetivo es analizar aspectos relevantes. En primer lugar se detalla la estructura de las publicaciones para proporcionar una visión de conjunto de su contenido. También se analiza si los heurísticos están orientados a dispositivos móviles y si incluyen una o varias checklists para facilitar la aplicación de las directrices a la evaluación de una web. Comprobar la existencia de checklists se estima oportuno porque se trata de una herramienta útil para el análisis de expertos, pero también porque en muchos casos constituyen un resumen práctico de las directrices.

Además de estos aspectos, Quiñones y Rusu (2017) señalan que muchos heurísticos se elaboran a partir de la experiencia y no como resultado de un proceso metodológico. Por eso, también se analiza si cada publicación incluye algún tipo de evidencia empírica que sustente los heurísticos, ya que es relevante saber si las directrices son el resultado de un proceso de investigación llevado a cabo por los propios autores (evidencia empírica primaria) o si, por el contrario, han sido confeccionadas por expertos en el área o elaboradas a partir de otros indicadores heurísticos (evidencia empírica secundaria).

Por último, la tabla también recoge el/los sistema/s de arquitectura de la información (Rosenfeld; Morville; Arango, 2015) que abarca cada protocolo. Esta información es especialmente relevante porque hace posible que los investigadores y profesionales que utilicen la taxonomía vislumbren el tipo de contenido que incorpora cada directriz.

\section{Resultados y conclusiones}

Tras realizar la revisión y el análisis de los protocolos más relevantes, se han obtenido dos taxonomías: la primera recoge los elementos formales (Anexo I) y la segunda la información sobre el contenido de los indicadores heurísticos (Anexo II).

Se analiza si cada publicación incluye algún tipo de evidencia empírica que sustente los heurísticos, ya que es relevante saber si las directrices son resultado de un proceso de investigación

\subsection{Elementos formales (Anexo I)}

La categorización en el apartado metodológico arroja cinco tipos de directrices en función de la naturaleza de su autoría, como se puede observar en el Anexo I: institucionales, gubernamentales, de expertos, online y científicas.

De las 44 publicaciones analizadas, 4 son institucionales, 11 de expertos, 13 gubernamentales, 4 online y 12 científicas. Las fuentes con mayor producción de indicadores heurísticos son, por tanto, las entidades gubernamentales, de investigadores y de expertos. Esto indica, por un lado, la creciente preocupación de los gobiernos por ofrecer webs de calidad a sus ciudadanos y, por otro, el interés en el mundo académico por este tema de estudio desde los años 90 .

En cuanto a la fecha de publicación, esta revisión ha permitido inferir que existen dos grandes etapas en la evolución de estos indicadores:

La primera se extiende de 1988 a 2006 y comprende el $22,72 \%$ de las publicaciones analizadas; incluye directrices sobre diseño de interfaces y software que no fueron explícitamente elaboradas para la web. Un aspecto transversal a estas publicaciones es su desactualización, pues con el paso 
de los años algunas han quedado superadas por trabajos posteriores y otras ya no están vigentes hoy en día. Aun así, han sido incluidas en el análisis por la trascendencia que han tenido desde su publicación.

La segunda etapa abarca de 2007 hasta la actualidad y comprende el $77,27 \%$ de las publicaciones. Se trata de principios directamente aplicables al momento actual de la Web que muestran una clara evolución respecto a las anteriores y se pueden aplicar con menor margen de error al análisis y evaluación de las interfaces actuales.

Hay cinco tipos de directrices en función de la naturaleza de su autoría: institucionales, gubernamentales, de expertos, online y científicas

Si bien es cierto que la producción de estos protocolos tiene origen en varios países (como se puede observar en el Anexo I, los documentos analizados fueron publicados por entidades o expertos procedentes de países de todo el mundo), resulta significativo que un gran número de las directrices analizadas fueron publicadas en EUA (34,09\%). Este resultado pone de manifiesto que la mayor parte de las directrices se enmarcan dentro de la cultura norteamericana (Al-Badi; Ali; Al-Balushi, 2012).

Respecto al tipo de publicación, los indicadores heurísticos analizados adoptan diferentes formatos: publicación online $(45,45 \%)$, libro o capítulo de libro (25\%), actas de congreso $(15,90 \%)$ y artículos científicos $(13,63 \%)$. Algunos de estos documentos están disponibles online y también en formato impreso, como es el caso de las normas ISO 9241-210:2010 e ISO 9241-151:2008, las HHS Guidelines de EUA y la propuesta del gobierno sueco.

\subsection{Elementos de contenido (Anexo II)}

Al analizar el enfoque móvil de los indicadores heurísticos, en la taxonomía que recoge el Anexo II se indican cuatro posibilidades:

- No: la publicación no contempla directrices dirigidas a estos dispositivos;

- Sí: las directrices abarcan todo tipo de dispositivos;

- Sí (sección específica): el documento le dedica una sección;

- Sí (nativo móvil): protocolo específicamente creado para smartphones y tablets.

El $50 \%$ de las directrices no están orientadas a móviles; del $50 \%$ restante, el $27,27 \%$ posee una aproximación general a los dispositivos móviles, el 18,18\% son indicadores específicamente elaborados para smartphones y tablets, y el $4,54 \%$ contiene una sección específica.

Resulta significativo que el $63,63 \%$ de las publicaciones analizadas no están basadas ni hacen referencia a ningún tipo de evidencia empírica; 9 de los indicadores (20,45\%) se basan en fuentes de investigación secundarias y sólo 8 $(18,18 \%)$ han realizado algún tipo de prueba científica para sustentar los heurísticos que contienen.
Hay que destacar que, dada su naturaleza científica, son los trabajos académicos los que más datos empíricos aportan. Resulta llamativo que la gran mayoría de expertos, organizaciones y entidades gubernamentales no basen sus directrices en la evidencia. Ante el avance constante de la tecnología y la gran variedad de dispositivos de acceso, realizar pruebas empíricas debería ser el método fundamental para extraer este tipo de recomendaciones. Además, los experimentos deberían limitarse a dominios concretos (no es lo mismo una web de e-commerce que una plataforma gubernamental) y establecer indicadores heurísticos específicos para cada uno de ellos.

\section{Los trabajos académicos son los que más datos empíricos aportan}

El análisis de los resultados contenidos en el Anexo II permite también comprobar que el $65,90 \%$ de las publicaciones incluyen algún tipo de checklist para recoger todos o algunos de los principios, algo que no es de extrañar, ya que el propósito de muchos de estos documentos es servir como base para la realización de evaluaciones heurísticas. Por otro lado, el 93,18\% de las publicaciones contiene heurísticos que forman parte de alguno de los sistemas de arquitectura de la información descritos por Rosenfeld, Morville y Arango (2015) y, concretamente, 15 documentos hacen referencia a los cuatro sistemas. Esta información aporta una nueva dimensión a estas publicaciones, y es valiosa para los profesionales y académicos que busquen indicadores heurísticos relacionados con algunos de los cuatro ámbitos.

En definitiva, ante la multiplicidad y heterogeneidad de indicadores heurísticos, este trabajo aporta una clasificación de las directrices más relevantes para facilitar a profesionales e investigadores su localización, análisis y aplicación.

Tras recopilar los indicadores heurísticos con mayor relevancia científica y geográfica, y analizar en profundidad cada publicación, el resultado son dos taxonomías (Anexos I y II) que estructuran los protocolos según diferentes elementos formales y de contenido. Ambas clasificaciones constituyen un instrumento que puede ser empleado por profesionales e investigadores para afrontar sus trabajos, permitiéndoles localizar, analizar y seleccionar las directrices que necesitan aplicar en cada caso y que, además, contribuye a estructurar y aportar orden a este tema.

\section{Nota}

Este trabajo forma parte de las investigaciones sobre el ciclo de la vida informativa y los formatos y narrativas digitales interactivas, objetivos Ob1 y Ob2 del proyecto Creación y contenido interactivo en la comunicación de información audiovisual: audiencias, diseño, sistemas y formatos CSO201564955-C4-2-R (Mineco/Feder), Ministerio de Economía y Competitividad (España).

\section{Referencias}

AIMC (2017). Audiencia de internet en el EGM.

http://www.aimc.es/-Audiencia-de-Internet-en-el-EGM-.html 
Al-Badi, Ali; Ali, Saqib; Al-Balushi, Taiseera (2012). "Ergonomics of usability/accessibility-ready websites: tools and guidelines". Webology, v. 9, n. 2, pp. 11-20.

http://www.webology.org/2012/v9n2/a98.html

Badashian, Ali-Sajedi; Mahdavi, Mehregan; PourShirMohammadi, Amir; Monajjemi-Nejad, Minoo (2008). "Fundamental usability guidelines for user interface design". En: Int conf on computational sciences and its applications, 2008 (ICCSA '08), pp. 106-113.

https://goo.gl/Kmvhf8

https://doi.org/10.1109/ICCSA.2008.45

Bevan, Nigel (2005). "Guidelines and standards for web usability". En: Proceedings $\mathrm{HCl}$ International 2005.

https://goo.gl/BYiEeg

Bevan, Nigel; Spinhof, Lonneke (2007). "Are guidelines and standards for web usability comprehensive?" In: Procs $\mathrm{HCl}$ International 2007.

https://goo.gl/bcHEkS

Brown, C. Marlin (1998). Human-computer interface design guidelines. Norwood: Ablex Publishing Corp. ISBN: 089391 3324

Carvajal, Mario; Saab, Juan (2010). Directrices de usabilidad para sitios web del Estado colombiano. Ministerio de Tecnologías de la Información y las Comunicaciones (República de Colombia).

http://www.mariocarvajal.com/Directrices-de-usabilidadpara-sitios-web-del-Estado-en-Colombia.pdf

Chuan, Ngip-Khean; Sivaji, Ashok; Wan-Ahmad, Wan-Fatimah (2014). "Proposed usability heuristics for testing gestural interaction". En: $4^{\text {th }}$ Intl conf on artificial intelligence with applications in engineering and technology (Icaiet), pp. 233-238.

https://doi.org/10.1109/ICAIET.2014.46

CIO, US Chief Information Officer (2015). US web design standards.

Codina, Lluís (2016). “Utilizar con éxito bases de datos académicas en tesis doctorales: el método facetar-derivar-combinar". Lluís Codina. Comunicación y documentación, 4 julio. https://www.lluiscodina.com/busqueda-bases-de-datosacademicas

Connors, Adam; Sullivan, Bryan (eds.) (2010). Mobile web application best practices. W3C.

https://www.w3.org/TR/mwabp

Constantine, Larry L. (1995). "What do users want? Engineering usability into software". Windows tech journal, v. 4, n. 12 , pp. 30-39.

De-Almeida-Pacheco, Beatriz; De-Almeida-Souza-Concilio, Ilana (2014). "Externalist philosophy and interaction: Proposal of heuristics for the design of interactive systems". En: Science and information conference (SAI) 2014, pp. 702-708. https://doi.org/10.1109/SAI.2014.6918264

Digital Transformation Office (2015). Digital service standard. Australian Government.

https://www.dto.gov.au/standard
Donker-Kuijer, Marieke-Welle; De-Jong, Menno; Lentz, Leo (2010). "Usable guidelines for usable websites? An analysis of five e-government heuristics". Government information quarterly, v. 27, n. 3, pp. 254-263.

https://doi.org/10.1016/j.giq.2010.02.006

European Commission (s.f.). Information providers guide. The EU internet handbook.

http://ec.europa.eu/ipg

García-López, Eva; García-Cabot, Antonio; Manresa-Yee, Cristina; De-Marcos, Luis; Pagés-Arévalo, Carmen (2017). "Validation of navigation guidelines for improving usability in the mobile web". Computer standards \& interfaces, v. 52, pp. 51-62. https://goo.gl/aa2muh https://doi.org/10.1016/j.csi.2017.01.011

Garreta-Domingo, Muriel; Mor-Pera, Enric (2010). Diseño centrado en el usuario. Universitat Oberta de Catalunya. https://goo.gl/68wpTe

Garrett, Jesse-James (2010). Elements of user experience. user-centered design for the Web and beyond. Berkeley: New Riders. ISBN: 9780321683687

Gobierno de Chile (2012). Guía digital. http://www.guiadigital.gob.cl/guia-web

Gob.UK (2015). Service manual.

https://www.gov.uk/service-manual

González, María-Paula; Lorés, Jesús; Granollers, Antoni (2008). "Enhancing usability testing through datamining techniques: A novel approach to detecting usability problem patterns for a context of use". Information and software technology, v. 50, n. 6, pp. 547-568.

https://goo.gl/v18dLd

https://doi.org/10.1016/j.infsof.2007.06.001

Google; Answer Lab (2016). Principles of mobile site design: delight users and drive conversions.

https://www.thinkwithgoogle.com/articles/principlesmobile-site-design-delight-users-drive-conversions.html

Government of India (2009). Guidelines for Indian government websites.

http://guidelines.gov.in

Grant, Maria J.; Booth, Andrew (2009). "A typology of reviews: an analysis of 14 review types and associated methodologies". Health information \& libraries journal, v. 26, n. 2, pp. 91-108. https://goo.gl/QGMs8t https://doi.org/10.1111/j.1471-1842.2009.00848.x

Hassan-Montero, Yusef (2013). Introducción a la interacción persona-ordenador. Universitat Oberta de Catalunya. https://goo.gl/xW7ELx

Hassan-Montero, Yusef; Martín-Fernández, Francisco J. (2003). "Guía de evaluación heurística de sitios web". No sólo usabilidad, 30 marzo.

http://www.nosolousabilidad.com/articulos/heuristica.htm

Henninger, Scott (2000). "A methodology and tools for applying context-specific usability guidelines to interface design". Interacting with computers, v. 12, n. 3, pp. 225-243. https://goo.gl/TsrfJG 
https://doi.org/10.1016/S0953-5438(99)00013-2

HHS; GSA (2006). Research-based web design \& usability guidelines. US Department of Health and Human Sciences; US General Services Administration. ISBN: 0160762707 https://goo.gl/A2Nsmn

Inostroza, Rodolfo; Rusu, Cristian; Roncagliolo, Silvana; Rusu, Virginica; Collazos, César A. (2016). "Developing Smash: A set of SMArtphone's uSability Heuristics". Computer standards \& interfaces, v. 43, pp. 40-52.

https://goo.gl/rrScrf

https://doi.org/10.1016/j.csi.2015.08.007

ISO (2008). Ergonomía de la interacción hombre-sistema. Parte 151: Directrices para las interfaces de usuario web (ISO 9241-151:2008).

http://www.aenor.es/aenor/normas/normas/fichanorma. asp ?tipo $=N \&$ codigo $=N 0042480 \#$.WRM4fcm_NbU

ISO (2010). Ergonomía de la interacción hombre-sistema. Parte 210: Diseño centrado en el operador humano para los sistemas interactivos (ISO 9241-210:2010).

http://www.aenor.es/aenor/normas/normas/fichanorma. asp?tipo $=N \&$ codigo $=N 0046310 \# . W R M 438 m \_N b U$

Joyce, Ger; Lilley, Mariana (2014). "Towards the development of usability heuristics for native smartphone mobile applications". En: Aaron, Marcus (ed.). Design, user experience, and usability. Theories, methods, and tools for designing the user experience. Proceedings Third int conf, DUXU 2014, v. 8517.

https://goo.gl/QTdfrr

https://doi.org/10.1007/978-3-319-07668-3_45

Krug, Steve (2014). Don't make me think revisited: A common sense approach to web and mobile usability. Thousand Oaks, CA: New Riders Publishing. ISBN: 9780321965516

Kuniavsky, Mike (2010). Smart things: Ubiquitous computing user experience design. Amsterdam: Morgan Kaufmann. ISBN: 9780123748997

Lobo, Desmond; Kaskaloglu, Kerem; Kim, Cha-Young; Herbert, Sandra (2011). "Web usability guidelines for smartphones: a synergic approach". En: International journal of information and electronics engineering, v. 1, n. 1, pp. 33. https://goo.gl/5q93Ga

Mariage, Céline; Vanderdonckt, Jean; Pribeanu, Costin (2005). "State of the art of web usability guidelines". The handbook of human factors in web design, pp. 688-700. https://goo.gl/i8rMcr

Mayhew, Deborah J. (1992). Principles and guidelines in software user interface design. New York: Prentice Hall, Inc. ISBN: 9780137219292

New Zealand Government (s.f.). Web usability standard 1.2. https://webtoolkit.govt.nz/standards/web-usabilitystandard-1-2

Nielsen, Jakob (1994). “Heuristic evaluation”. En: Nielsen, Jakob; Mack, Robert L. (eds.). Usability inspection methods. New York: John Wiley \& Sons. ISBN: 0471018775

Nielsen, Jakob; Molich, Rolf (1990). "Heuristic evaluation of user interfaces". En: Proceedings of the Sigchi conf on hu- man factors in computing systems, pp. 249-256.

https://goo.gl/TemKqv

Nielsen, Jakob; Tahir, Marie (2002). Usabilidad de páginas de inicio: análisis de 50 sitios web. Madrid: Pearson. ISBN: 9788420532028

Norman, Don (1988). The design of everyday things. New York: Basic Books Publishers Inc. ISBN: 9780465050659

Office of the e-Envoy Cabinet Office (2003). Guidelines for UK government websites: illustrated handbook for web management teams. ISBN: 9780114301798

Ohnemus, Kenneth R. (1997). "Web style guides: who, what, where". En: Proceedings of the $15^{\text {th }}$ Annual int conf on computer documentation, Sigdoc'97, pp. 189-197. https://doi.org/10.1145/263367.263392

Pedraza-Jiménez, Rafael; Codina, Lluís; Guallar, Javier (2016). Calidad en sitios web: método de análisis general, e-commerce, imágenes, hemerotecas y turismo. Barcelona: Editorial UOC. ISBN: 9788490644874

Pereira-Da-Silva, Deógenes; De-Souza, Patricia-Cristiane; Maciel, Cristiano (2016). "Establishing guidelines for user quality of experience in ubiquitous systems". En: Int/ conf on distributed, ambient, and pervasive interactions (pp. 46-57). https://doi.org/10.1007/978-3-319-39862-4_5

Quiñones, Daniela; Rusu, Cristian (2017). “How to develop usability heuristics: A systematic literature review". Computer standards \& interfaces, v. 53, pp. 89-122.

https://goo.gl/rC9ptp

http://dx.doi.org/10.1016/j.csi.2017.03.009

Rabin, Jo; McCathieNevile, Charles (2008). Mobile web best practices 1.0. Basic guidelines.

https://www.w3.org/TR/mobile-bp/\#d0e1733

Rosenfeld, Louis; Morville, Peter; Arango, Jorge (2015). Information architecture for the web and beyond. Sebastopol, CA: O’Reilly Media Inc. ISBN: 9781491911686

Shneiderman, Ben; Plaisant, Catherine (1987). “Designing the user interface: Strategies for effective human-computer interaction". ACM Sigbio newsletter, v. 9, n. 1.

Shneiderman, Ben; Plaisant, Catherine; Cohen, Maxine; Jacobs, Steven; Elmqvist, Niklas; Diakopoulos, Nicholas (2016). Designing the user interface: Strategies for effective human-computer interaction. Sixth Edition. Pearson. ISBN: 9780134380834

Smith, Sidney; Mosier, Jane (1986). Guidelines for designing user interface software. Bedford, MA: Mitre corp. ISBN: 978 9992080412

Suárez-Torrente, M. Carmen; Martínez-Prieto, A. Belén; Álvarez-Gutiérrez, Darío; Alva-De-Sagastegui, M. Elena (2013). "Sirius: A heuristic-based framework for measuring web usability adapted to the type of website". Journal of systems and software, v. 86, n. 3, pp. 649-663.

https://doi.org/10.1016/j.jss.2012.10.049

Sutcliffe, Alistair (2002). "Assessing the reliability of heuristic evaluation for web site attractiveness and usability". En: Conf on system sciences. HICSS. Proceedings of the $35^{\text {th }} \mathrm{An}$ - 
nual Hawaii international, pp. 1838-1847.

Tasmanian Government (2010a). Web design and navigation guidelines. Office of eGovernment, Tasmanian Government. https://goo.gl/i1v2Wa

Tasmanian Government (2010b). Web usability guidelines. Office of eGovernment, Tasmanian Government. http://www.egovernment.tas.gov.au/standards_and_guidelines

Tognazzini, Bruce (2003). "First principles of interaction design". Ask Tog. Interaction design solutions for the real world. http://asktog.com/atc/principles-of-interaction-design

Tognazzini, Bruce (2014). "First principles of interaction design (revised \& expanded)". Ask Tog. Interaction design solutions for the real world, 5 March.

http://asktog.com/atc/principles-of-interaction-design

Toxboe, Anders (2011). "User interface design pattern library". UI Patterns.

http://ui-patterns.com/patterns

UXPA (2011). "Principles for usable design". Usability body of knowledge. Usability Professionals' Association.

http://www.usabilitybok.org/principles-for-usable-design https://standards.usa.gov

Väänänen-Vainio-Mattila, Kaisa; Wäljas, Minna (2009). "Development of evaluation heuristics for web service user experience". In: CHI'09 Extended abstracts on human factors in computing systems, pp. 3679-3684.

https://goo.gl/nwF4nV

Van-Welie, Martijn (2008). "Pattern library". Welie.com. Patterns in interaction design.

http://www.welie.com/patterns

Verva (2006). Swedish national guidelines for public sector websites.

Wright, Peter; Blythe, Mark (2007). “User experience research as an inter-discipline: Towards a UX manifesto". En: Law, Effie; Vermeeren, Arnold; Hassenzahl, Marc; Blythe, Mark (eds). Proceedings of the Workshop on towards a UX manifesto, pp. 65-70. https://goo.gl/fSPXe3

Yáñez-Gómez, Rosa; Cascado-Caballero, Daniel; Sevillano, José-Luis (2014). "Heuristic evaluation on mobile interfaces: A new checklist". The scientific world journal, v. 2014. https://doi.org/10.1155/2014/434326

Anexo I. Elementos formales

\begin{tabular}{|c|c|c|c|c|}
\hline & Autor & Año & País & $\begin{array}{l}\text { Tipo de } \\
\text { publicación }\end{array}$ \\
\hline \multicolumn{5}{|l|}{ Directrices institucionales } \\
\hline Mobile web best practices 1.0 & Rabin; McCathieNevile (eds.) & 2008 & EUA & Online \\
\hline Mobile web application best practices & Connors; Sullivan (eds.) & 2010 & EUA & Online \\
\hline $\begin{array}{l}\text { Ergonomics of human-system interaction - Part 210: Human-centred } \\
\text { design for interactive systems (ISO 9241-210:2010) }\end{array}$ & ISO & 2010 & Suiza & Norma ISO \\
\hline $\begin{array}{l}\text { Ergonomics of human-system interaction - Part 151: Guidance on } \\
\text { world wide web user interfaces (ISO 9241-151:2008) }\end{array}$ & ISO & 2008 & Suiza & Norma ISO \\
\hline \multicolumn{5}{|l|}{ Directrices gubernamentales } \\
\hline Research-based web design \& usability guidelines & $\begin{array}{l}\text { US Department of Health and } \\
\text { Human Sciences (HSS) }\end{array}$ & 2006 & EUA & Libro y online \\
\hline US web design standards & $\mathrm{ClO}$ & 2015 & EUA & Online \\
\hline Illustrated handbook for web management teams & $\begin{array}{l}\text { Office of the e-Envoy Cabinet } \\
\text { Office }\end{array}$ & 2003 & Reino Unido & Manual \\
\hline Service manual & $\begin{array}{l}\text { Government Digital Service } \\
\text { (Gob.UK) }\end{array}$ & 2016 & Reino Unido & Online \\
\hline Digital service standard & Digital Transformation Office & 2015 & Australia & Online \\
\hline Guía digital & Gobierno de Chile & 2012 & Chile & Online \\
\hline Swedish national guidelines for public sector websites & Verva & 2006 & Suecia & Libro y online \\
\hline Web design and navigation guidelines & Tasmanian Government & 2010 & Tasmania & Online \\
\hline Web usability guidelines & Tasmanian Government & 2010 & Tasmania & Online \\
\hline Directrices de usabilidad para sitios web del Estado colombiano & Carvajal; Saab & 2010 & Colombia & Online \\
\hline Web usability standard 1.2 & New Zealand Government & 2013 & Nueva Zelanda & Online \\
\hline
\end{tabular}




\begin{tabular}{|c|c|c|c|c|}
\hline Guidelines for Indian Government websites & Government of India & 2009 & India & Online \\
\hline Information providers guide & European Commission & s.f. & UE & Online \\
\hline \multicolumn{5}{|l|}{ Directrices de expertos } \\
\hline 10 Usability heuristics for user interface design & Nielsen & 1994 & EUA & Capítulo de libro \\
\hline The eight golden rules of interface design & Shneiderman et al. & 2010 & EUA & Libro \\
\hline First principles of interaction design & Tognazzini & 2014 & EUA & Online \\
\hline $\begin{array}{l}\text { Don't make me think (revisited). A common sense approach to web } \\
\text { and mobile usability }\end{array}$ & Krug & 2014 & EUA & Libro \\
\hline Principios de Deborah Mayhew & Mayhew & 1992 & EUA & Libro \\
\hline Information architecture for the web and beyond & Rosenfeld; Morville; Arango & 2015 & EUA & Libro \\
\hline Guía de evaluación heurística de sitios web & $\begin{array}{l}\text { Hassan-Montero; Matín- } \\
\text { Fernández }\end{array}$ & 2003 & España & Online \\
\hline What do users want? Engineering usability into software & Constantine & 1995 & Australia & Artículo científico \\
\hline $\begin{array}{l}\text { Seven principles for transforming difficult tasks into simple ones } \\
\text { (user-centred design) }\end{array}$ & Norman & 1988 & EUA & Libro \\
\hline Human-computer interface design guidelines & Brown & 1998 & EUA & Libro \\
\hline Sistema articulado de análisis de medios digitales (Saamd) & $\begin{array}{l}\text { Pedraza-Jiménez; Codina; } \\
\text { Guallar }\end{array}$ & 2016 & España & Libro \\
\hline \multicolumn{5}{|l|}{ Directrices online } \\
\hline Principles for usable design & UXPA & 2011 & EUA & Online \\
\hline Patterns in interaction design (pattern library) & Van-Welie & 2008 & Holanda & Online \\
\hline User interface design pattern library & Toxboe & 2011 & Dinamarca & Online \\
\hline Principales of mobile site design & Google; Answer Lab & s.f. & EUA & Online \\
\hline \multicolumn{5}{|l|}{ Directrices científicas } \\
\hline Heuristic evaluation on mobile interfaces: a new checklist & $\begin{array}{l}\text { Yáñez-Gómez; Cascado-Ca- } \\
\text { ballero; Sevillano }\end{array}$ & 2014 & España & Artículo científico \\
\hline Developing Smash: a set of SMArtphone's uSability Heuristics & Inostroza et al. & 2016 & $\begin{array}{l}\text { Chile y Colom- } \\
\text { bia }\end{array}$ & Artículo científico \\
\hline Web usability guidelines for smartphones: a synergic approach & Lobo et al. & 2011 & Desconocido & Artículo científico \\
\hline Fundamental usability guidelines for user interface design & Badashian et al. & 2008 & Irán & $\begin{array}{l}\text { Comunicación } \\
\text { congreso }\end{array}$ \\
\hline $\begin{array}{l}\text { Sirius: a heuristic-based framework for measuring web usability } \\
\text { adapted to the type of website }\end{array}$ & Suárez-Torrente et al. & 2013 & España & Artículo científico \\
\hline Proposed usability heuristics for testing gestural interaction & Chuan; Sivaji; Ahmad & 2014 & Malaysia & $\begin{array}{l}\text { Comunicación } \\
\text { congreso }\end{array}$ \\
\hline $\begin{array}{l}\text { Assessing the reliability of heuristic evaluation for website attractive- } \\
\text { ness and usability }\end{array}$ & Sutcliffe & 2002 & Reino Unido & $\begin{array}{l}\text { Comunicación } \\
\text { congreso }\end{array}$ \\
\hline Development of evaluation heuristics for web service user experience & $\begin{array}{l}\text { Väänänen-Vainio-Mattila; } \\
\text { Wäljas }\end{array}$ & 2009 & Finlandia & $\begin{array}{l}\text { Comunicación } \\
\text { congreso }\end{array}$ \\
\hline $\begin{array}{l}\text { Establishing guidelines for user quality of experience in ubiquitous } \\
\text { systems }\end{array}$ & $\begin{array}{l}\text { Pereira-Da-Silva; De-Souza; } \\
\text { Maciel }\end{array}$ & 2016 & Brasil & $\begin{array}{l}\text { Comunicación } \\
\text { congreso }\end{array}$ \\
\hline $\begin{array}{l}\text { Externalist philosophy and interaction: proposal of heuristics for the } \\
\text { design of interactive systems }\end{array}$ & $\begin{array}{l}\text { De-Almeida Pacheco; De } \\
\text { Almeida-Souza-Concilio }\end{array}$ & 2014 & Brasil & $\begin{array}{l}\text { Comunicación } \\
\text { congreso }\end{array}$ \\
\hline $\begin{array}{l}\text { Towards the development of usability heuristics for native smart- } \\
\text { phone mobile applications }\end{array}$ & Joyce; Lilley & 2014 & Malaysia & $\begin{array}{l}\text { Comunicación } \\
\text { congreso }\end{array}$ \\
\hline $\begin{array}{l}\text { Validation of navigation guidelines for improving usability in the } \\
\text { mobile web }\end{array}$ & García-López et al. & 2017 & España & Artículo científico \\
\hline
\end{tabular}


Anexo II. Elementos de contenido

\begin{tabular}{|c|c|c|c|c|c|c|}
\hline & Estructura & Móviles & $\begin{array}{l}\text { Evidencia } \\
\text { empírica } \\
\text { primaria }\end{array}$ & $\begin{array}{l}\text { Evidencia } \\
\text { empírica } \\
\text { secundaria }\end{array}$ & $\begin{array}{l}\text { Chec- } \\
\text { klist }\end{array}$ & $\begin{array}{l}\text { Sistemas ar- } \\
\text { quitectónicos }\end{array}$ \\
\hline \multicolumn{7}{|l|}{ Directrices institucionales } \\
\hline Mobile web best practices 1.0 & $\begin{array}{l}\text { Overall behavior } \\
\text { Navigation and links } \\
\text { Page layout and content } \\
\text { Page definition } \\
\text { User input }\end{array}$ & $\begin{array}{l}\text { Sí } \\
\text { (nativo } \\
\text { móvil) }\end{array}$ & No & No & Sí & $\begin{array}{l}\text { Organización, } \\
\text { etiquetado y } \\
\text { navegación }\end{array}$ \\
\hline $\begin{array}{l}\text { Mobile web application best } \\
\text { practices }\end{array}$ & $\begin{array}{l}\text { Application data } \\
\text { Security and privacy } \\
\text { User awareness and control } \\
\text { Conservative use of resources } \\
\text { User experience } \\
\text { Handling variations in the delivery context } \\
\text { Further considerations }\end{array}$ & $\begin{array}{c}\text { Sí } \\
\text { (nativo } \\
\text { móvil) }\end{array}$ & No & No & Sí & $\begin{array}{l}\text { Organización y } \\
\text { navegación }\end{array}$ \\
\hline $\begin{array}{l}\text { Ergonomics of human-system } \\
\text { interaction - Part 210: human-cen- } \\
\text { tred design for interactive systems } \\
\text { (ISO 9241-210:2010) }\end{array}$ & $\begin{array}{l}\text { Principles of human-centred design } \\
\text { Planning human-centred design } \\
\text { Human-centred design activities }\end{array}$ & No & No & No & Sí & Navegación \\
\hline $\begin{array}{l}\text { Ergonomics of human-system } \\
\text { interaction - Part 151: guidance on } \\
\text { World Wide Web user interfaces } \\
\text { (ISO 9241-151:2008) }\end{array}$ & $\begin{array}{l}\text { Decisiones en el diseño de alto nivel y estrate- } \\
\text { gia de diseño } \\
\text { Diseño de contenido } \\
\text { Navegación y búsqueda } \\
\text { Presentación del contenido } \\
\text { Aspectos generales del diseño }\end{array}$ & No & No & No & Sí & $\begin{array}{l}\text { Organización, } \\
\text { etiquetado, } \\
\text { navegación y } \\
\text { búsqueda }\end{array}$ \\
\hline \multicolumn{7}{|l|}{ Directrices gubernamentales } \\
\hline $\begin{array}{l}\text { Research-based web design \& } \\
\text { usability guidelines }\end{array}$ & $\begin{array}{l}\text { Design process and evaluation } \\
\text { Optimizing the user experience } \\
\text { Accessibility } \\
\text { Hardware and software } \\
\text { The homepage } \\
\text { Page layout } \\
\text { Navigation } \\
\text { Scrolling and paging } \\
\text { Heading, titles, and labels } \\
\text { Links } \\
\text { Text appearance } \\
\text { Lists } \\
\text { Screen-based controls } \\
\text { Graphics, images, and multimedia } \\
\text { Writing web content } \\
\text { Content organization } \\
\text { Search } \\
\text { Usability testing }\end{array}$ & No & No & Sí & Sí & $\begin{array}{l}\text { Organización, } \\
\text { etiquetado, } \\
\text { navegación y } \\
\text { búsqueda }\end{array}$ \\
\hline US web design standards & $\begin{array}{l}\text { Typography } \\
\text { Colors } \\
\text { Accessibility } \\
\text { Grids } \\
\text { Buttons } \\
\text { Labels } \\
\text { Tables } \\
\text { Alerts } \\
\text { Accordions } \\
\text { Form controls } \\
\text { Form templates } \\
\text { Search bar } \\
\text { Side navigation } \\
\text { Headers } \\
\text { Footers }\end{array}$ & No & Sí & No & No & $\begin{array}{l}\text { Organización, } \\
\text { etiquetado, } \\
\text { navegación y } \\
\text { búsqueda }\end{array}$ \\
\hline $\begin{array}{l}\text { Illustrated handbook for web } \\
\text { management teams }\end{array}$ & $\begin{array}{l}\text { Website managemente } \\
\text { Content of websites } \\
\text { File storage and structure } \\
\text { HMTL markup, other formats and scripting } \\
\text { Developments } \\
\text { Technical detail and tutorials }\end{array}$ & No & No & No & $\begin{array}{l}\text { Sí } \\
\text { (algunas } \\
\text { seccio- } \\
\text { nes) }\end{array}$ & $\begin{array}{l}\text { Organización, } \\
\text { etiquetado y } \\
\text { navegación }\end{array}$ \\
\hline
\end{tabular}




\begin{tabular}{|c|c|c|c|c|c|c|}
\hline Service manual & $\begin{array}{l}\text { Agile delivery } \\
\text { Design } \\
\text { Helping people to use your service } \\
\text { Measuring success } \\
\text { Service assessments } \\
\text { Technology } \\
\text { The team } \\
\text { User research }\end{array}$ & Sí & Sí & No & No & $\begin{array}{l}\text { Organización, } \\
\text { etiquetado y } \\
\text { navegación }\end{array}$ \\
\hline Digital service standard & $\begin{array}{l}\text { Scope of Standard } \\
\text { Moratorium } \\
\text { Meeting the standard } \\
\text { Assessment reports } \\
\text { Design principles } \\
\text { Service design and delivery process } \\
\text { Guides } \\
\text { Common solutions } \\
\text { Glossary }\end{array}$ & Sí & No & No & No & $\begin{array}{c}\text { Organización, } \\
\text { etiquetado y } \\
\text { navegación }\end{array}$ \\
\hline Guía digital & $\begin{array}{l}\text { Normativas } \\
\text { Diseño de interfaces e interacción } \\
\text { Estándares web } \\
\text { Encontrabilidad } \\
\text { Usabilidad } \\
\text { Planificación de un sitio web } \\
\text { Puesta en marcha de sitio web } \\
\text { Mantención y operación del sitio } \\
\text { Checklist } \\
\text { Medición de resultados en redes sociales }\end{array}$ & No & No & No & $\begin{array}{l}\text { Sí (Chec- } \\
\text { klist Usa- } \\
\text { bilidad) }\end{array}$ & $\begin{array}{c}\text { Organización, } \\
\text { etiquetado y } \\
\text { navegación }\end{array}$ \\
\hline $\begin{array}{l}\text { Swedish national guidelines for } \\
\text { public sector websites }\end{array}$ & $\begin{array}{l}\text { Better and more efficient service } \\
\text { Development process } \\
\text { Website standards } \\
\text { Basic content and services } \\
\text { Keeping the website up to date } \\
\text { Web content for mobile devices } \\
\text { Web publishing tools } \\
\text { Assistive technologies for using websites }\end{array}$ & $\begin{array}{l}\text { Sí (sección } \\
\text { específica) }\end{array}$ & No & No & $\begin{array}{c}\text { Sí } \\
\text { (algunas } \\
\text { seccio- } \\
\text { nes) }\end{array}$ & $\begin{array}{c}\text { Organización, } \\
\text { etiquetado, } \\
\text { navegación y } \\
\text { búsqueda }\end{array}$ \\
\hline $\begin{array}{l}\text { Web design and navigation } \\
\text { guidelines }\end{array}$ & $\begin{array}{l}\text { Introduction } \\
\text { Header and footer elements } \\
\text { Page elements }\end{array}$ & No & No & No & No & $\begin{array}{l}\text { Etiquetado, } \\
\text { navegación y } \\
\text { búsqueda }\end{array}$ \\
\hline Web usability guidelines & $\begin{array}{l}\text { Policy context } \\
\text { Introduction to usability } \\
\text { Understanding the Characteristics of Web } \\
\text { Usage } \\
\text { User-centred website design } \\
\text { Best Practice in Web Design } \\
\text { Tools and resources } \\
\text { Links to further information }\end{array}$ & No & No & No & No & $\begin{array}{l}\text { Etiquetado y } \\
\text { navegación }\end{array}$ \\
\hline $\begin{array}{l}\text { Directrices de usabilidad para } \\
\text { sitios web del Estado colombiano }\end{array}$ & $\begin{array}{l}\text { Arquitectura de información } \\
\text { Diseño de interfaz de usuario } \\
\text { Diseño de interacción } \\
\text { Búsqueda } \\
\text { Pruebas de usabilidad } \\
\text { Contenido }\end{array}$ & No & No & Sí & No & $\begin{array}{l}\text { Organización, } \\
\text { etiquetado y } \\
\text { navegación }\end{array}$ \\
\hline Web usability standard 1.2 & $\begin{array}{l}\text { Application } \\
\text { Requirements (home page, contact informa- } \\
\text { tion, copyright, privacy, links to non-HTML } \\
\text { files, printable webpages) } \\
\text { Glossary }\end{array}$ & No & No & No & No & Navegación \\
\hline $\begin{array}{l}\text { Guidelines for Indian Government } \\
\text { websites }\end{array}$ & $\begin{array}{l}\text { Government of India Identifiers } \\
\text { Building Confidence } \\
\text { Scope of Content } \\
\text { Quality of Content } \\
\text { Design } \\
\text { Development } \\
\text { Website Hosting } \\
\text { Website Management }\end{array}$ & No & No & No & Sí & $\begin{array}{c}\text { Organización, } \\
\text { etiquetado, } \\
\text { navegación y } \\
\text { búsqueda }\end{array}$ \\
\hline Information providers guide & $\begin{array}{l}\text { Basics } \\
\text { Plan } \\
\text { Content } \\
\text { Design } \\
\text { Build } \\
\text { Go live } \\
\text { Maintain }\end{array}$ & Sí & No & No & Sí & $\begin{array}{c}\text { Organización, } \\
\text { etiquetado, } \\
\text { navegación y } \\
\text { búsqueda }\end{array}$ \\
\hline
\end{tabular}


Directrices de expertos interface design

1. Visibility of system status

2. Match between system and the real world

3. User control and freedom

4. Consistency and standards

5. Error prevention

6. Recognition rather than recall

7.Flexibility and efficiency of use

8. Aesthetic and minimalist design

9. Help users recognize, diagnose, and recover from errors

10. Help and documentation

1. Strive for consistency

2. Cater to universal usability.

3. Offer informative feedback.

The eight golden rules of interface design

4. Design dialogs to yield closure

5. Prevent errors

6. Permit easy reversal of actions

7. Support internal locus of control

8. Reduce short-term memory load

Aesthetics

Anticipation

Autonomy

Color

Consistency

Defaults

Discoverability

Efficiency of the user

First principles of interaction

Explorable interfaces

design

Fitts's law

Human interface objects

Latency reduction

Learnability

Use of metaphors

Protect users' work

Readability

Simplicity

State

Visible navigation

Don't make me think (revisited). A common sense approach to web

and mobile usability

Guiding principles

Things you need to get right

Making sure you got them right

Larger concerns and outside influences

Compatibilidad de usuario, de producto, de

tareas y de procesos

Consistencia y robustez

Familiaridad

Simplicidad

Manipulación directa

Principios de Deborah Mayhew

Control

Wysiwyg4

Flexibilidad

Sensibilidad y retroalimentación

Tecnología invisible

Protección

Facilidad de aprendizaje y facilidad de uso

Information architecture for the

Introducing information architecture

Basic principles of information architecture

web and beyond

Getting information architecture done

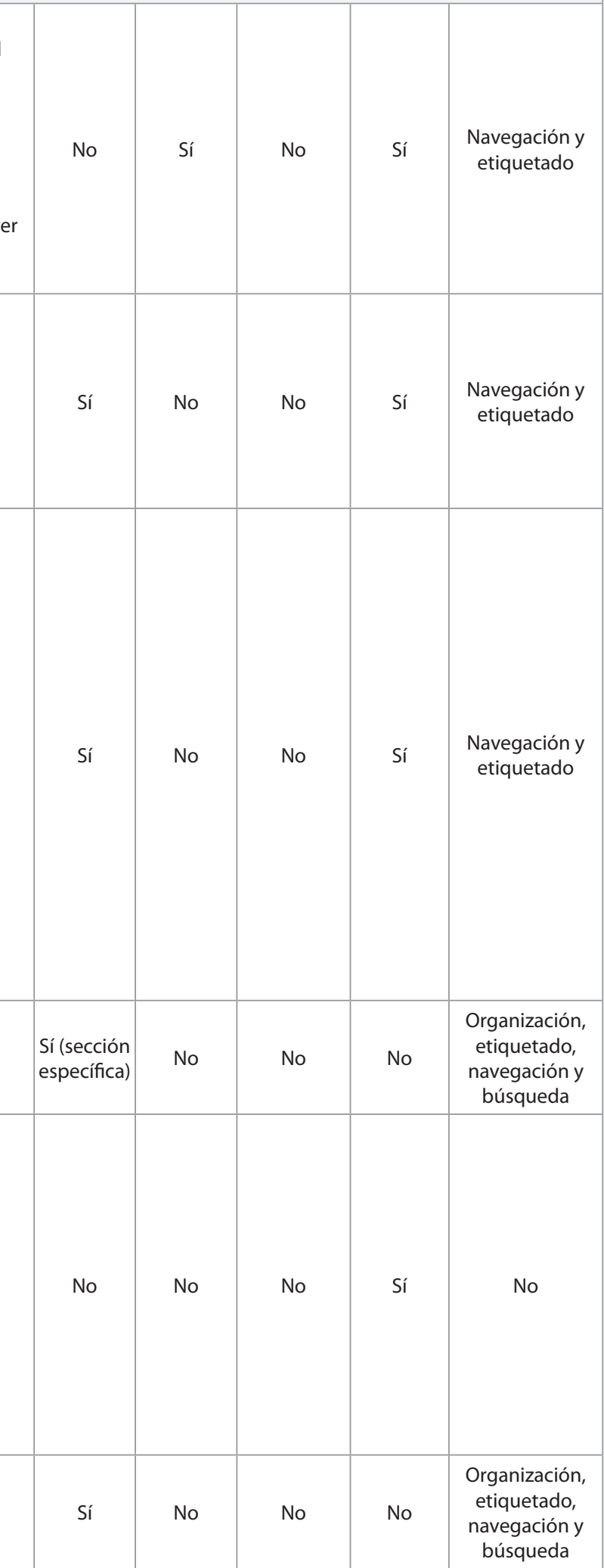




\begin{tabular}{|c|c|c|c|c|c|c|}
\hline $\begin{array}{l}\text { Guía de evaluación heurística de } \\
\text { sitios web }\end{array}$ & $\begin{array}{l}\text { Generales } \\
\text { Identidad e información } \\
\text { Lenguaje y redacción } \\
\text { Rotulado } \\
\text { Estructura y navegación } \\
\text { Lay-out de la página } \\
\text { Búsqueda } \\
\text { Elementos multimedia } \\
\text { Ayuda } \\
\text { Accesibilidad } \\
\text { Control y retroalimentación }\end{array}$ & No & No & No & Sí & $\begin{array}{l}\text { Organización, } \\
\text { etiquetado, } \\
\text { navegación y } \\
\text { búsqueda }\end{array}$ \\
\hline $\begin{array}{l}\text { What do users want? engineering } \\
\text { usability into software }\end{array}$ & $\begin{array}{l}\text { First rule: Access } \\
\text { Second rule: Efficacy } \\
\text { Third rule: Progression } \\
\text { Fourth rule: Support } \\
\text { Fifth rule: Context } \\
\text { Structure principle } \\
\text { Simplicity principle } \\
\text { Visibility principle } \\
\text { Reuse principle } \\
\text { Feedback principle } \\
\text { Tolerance principle }\end{array}$ & No & No & No & Sí & $\begin{array}{l}\text { Organización, } \\
\text { navegación y } \\
\text { etiquetado }\end{array}$ \\
\hline $\begin{array}{l}\text { Seven principles for transforming } \\
\text { difficult tasks into simple ones } \\
\text { (user-centred design) }\end{array}$ & $\begin{array}{l}\text { Use both knowledge in the world and knowle- } \\
\text { dge in the head } \\
\text { Simplify the structure of tasks } \\
\text { Make things visible: bridge the gulfs execution } \\
\text { and evaluation } \\
\text { Get the mappings right } \\
\text { Exploit the power of constraints, both natural } \\
\text { and artificial } \\
\text { Design for error } \\
\text { When all else fails, standardize }\end{array}$ & No & No & No & No & No \\
\hline $\begin{array}{l}\text { Human-computer interface design } \\
\text { guidelines }\end{array}$ & $\begin{array}{l}\text { Designing display formats } \\
\text { Effective wording } \\
\text { Color } \\
\text { Graphics } \\
\text { Dialogue design } \\
\text { Data entry } \\
\text { Control and display devices } \\
\text { Error messages and online assistance } \\
\text { Implementation of human-computer interface } \\
\text { Guidelines }\end{array}$ & No & No & No & Sí & $\begin{array}{l}\text { Organización, } \\
\text { etiquetado, } \\
\text { navegación y } \\
\text { búsqueda }\end{array}$ \\
\hline $\begin{array}{l}\text { Sistema articulado de análisis de } \\
\text { medios digitales (Saamd) }\end{array}$ & $\begin{array}{l}\text { Análisis qué queremos estudiar } \\
\text { Dimensiones } \\
\text { Parámetros } \\
\text { Preguntas de chequeo Indicadores } \\
\text { Tabla de análisis Aplicación y tabulación de } \\
\text { resultados }\end{array}$ & Sí & Sí & No & No & No \\
\hline \multicolumn{7}{|l|}{ Directrices online } \\
\hline Principles for usable design & $\begin{array}{l}\text { Usefulness } \\
\text { Consistency } \\
\text { Simplicity } \\
\text { Communication } \\
\text { Error prevention and handling } \\
\text { Efficiency } \\
\text { Workload reduction } \\
\text { Usability judgement }\end{array}$ & No & No & Sí & Sí & $\begin{array}{l}\text { Organización, } \\
\text { etiquetado y } \\
\text { navegación }\end{array}$ \\
\hline $\begin{array}{l}\text { Patterns in interaction design } \\
\text { (pattern library) }\end{array}$ & $\begin{array}{l}\text { Navigation around } \\
\text { Basic interactions } \\
\text { Searching } \\
\text { Dealing with data } \\
\text { Personalizing } \\
\text { Shopping } \\
\text { Making choices } \\
\text { Giving input } \\
\text { Miscellaneous }\end{array}$ & No & No & No & Sí & $\begin{array}{l}\text { Organización, } \\
\text { etiquetado, } \\
\text { navegación y } \\
\text { búsqueda }\end{array}$ \\
\hline
\end{tabular}




\begin{tabular}{|c|c|c|c|c|c|c|}
\hline $\begin{array}{l}\text { User interface design pattern } \\
\text { library }\end{array}$ & $\begin{array}{l}\text { Getting input } \\
\text { Navigation } \\
\text { Dealing with data } \\
\text { Social } \\
\text { Miscellaneous } \\
\text { Onboarding }\end{array}$ & Sí & No & No & Sí & $\begin{array}{l}\text { Organización, } \\
\text { etiquetado, } \\
\text { navegación y } \\
\text { búsqueda }\end{array}$ \\
\hline Principles of mobile site design & $\begin{array}{l}\text { Home page \& site navigation } \\
\text { Site search } \\
\text { Commerce \& conversions } \\
\text { Form entry } \\
\text { Usability \& Form factor } \\
\text { technical checklist }\end{array}$ & $\begin{array}{l}\text { Sí (nativo } \\
\text { móvil) }\end{array}$ & Sí & No & Sí & $\begin{array}{l}\text { Organización, } \\
\text { etiquetado, } \\
\text { navegación y } \\
\text { búsqueda }\end{array}$ \\
\hline \multicolumn{7}{|l|}{ Directrices científicas } \\
\hline $\begin{array}{l}\text { Heuristic evaluation on mobile } \\
\text { interfaces: a new checklist }\end{array}$ & $\begin{array}{l}\text { Visibility of system status } \\
\text { Match between system and the real world } \\
\text { (mental model accuracy) } \\
\text { User control } \\
\text { Consistency } \\
\text { Error prevention } \\
\text { Recognition rather than recall } \\
\text { Flexibility and efficiency of use } \\
\text { Aesthetic and minimalist design } \\
\text { Help users recognize, diagnose and recover } \\
\text { from errors } \\
\text { Help and documentation } \\
\text { Skills } \\
\text { Pleasurable and respectful interaction } \\
\text { Privacy }\end{array}$ & $\begin{array}{l}\text { Sí (nativo } \\
\text { móvil) }\end{array}$ & No & Sí & Sí & $\begin{array}{l}\text { Organización, } \\
\text { etiquetado, } \\
\text { navegación y } \\
\text { búsqueda }\end{array}$ \\
\hline $\begin{array}{l}\text { Developing Smash: a set of } \\
\text { SMArtphone's uSability Heuristics }\end{array}$ & $\begin{array}{l}\text { Visibility of system status } \\
\text { Match between system and the real world } \\
\text { User control and freedom } \\
\text { Consistency and standards } \\
\text { Error prevention } \\
\text { Minimize the user's memory load } \\
\text { Customization and shortcuts } \\
\text { Efficiency of use and performance } \\
\text { Aesthetic and minimalist } \\
\text { design } \\
\text { Help users recognize, diagnose, and recover } \\
\text { from errors } \\
\text { Help and documentation } \\
\text { Physical interaction and ergonomics }\end{array}$ & $\begin{array}{l}\text { Sí (nativo } \\
\text { móvil) }\end{array}$ & No & Sí & Sí & $\begin{array}{l}\text { Organización, } \\
\text { etiquetado y } \\
\text { navegación }\end{array}$ \\
\hline $\begin{array}{l}\text { Web usability guidelines for smar- } \\
\text { tphones: A synergic approach }\end{array}$ & $\begin{array}{l}\text { Keep it simple } \\
\text { Simplify user input } \\
\text { Scroll vertically only } \\
\text { Multiple versions of the website } \\
\text { Native apps versus mobile webs } \\
\text { Avoid repeating navigation }\end{array}$ & $\begin{array}{l}\text { Sí (nativo } \\
\text { móvil) }\end{array}$ & No & Sí & Sí & $\begin{array}{l}\text { Navegación y } \\
\text { búsqueda }\end{array}$ \\
\hline $\begin{array}{l}\text { Fundamental usability guidelines } \\
\text { for user interface design }\end{array}$ & $\begin{array}{l}\text { Access control } \\
\text { User control and freedom } \\
\text { Minimize the user's memory load } \\
\text { Creating multilingual forms } \\
\text { Minimalist design and aesthetic } \\
\text { Error prevention / handling } \\
\text { Anticipation }\end{array}$ & No & No & No & No & $\begin{array}{l}\text { Organización y } \\
\text { navegación }\end{array}$ \\
\hline $\begin{array}{l}\text { Sirius: a heuristic-based framework } \\
\text { for measuring web usability adap- } \\
\text { ted to the type of website }\end{array}$ & $\begin{array}{l}\text { General aspects } \\
\text { Identity and information } \\
\text { Structure and navigation } \\
\text { Labelling } \\
\text { Layout of the page } \\
\text { Comprehensibility and ease of interaction } \\
\text { Control and feedback } \\
\text { Multimedia elements } \\
\text { Search } \\
\text { Help }\end{array}$ & No & No & Sí & Sí & $\begin{array}{l}\text { Organización, } \\
\text { etiquetado, } \\
\text { navegación y } \\
\text { búsqueda }\end{array}$ \\
\hline $\begin{array}{l}\text { Proposed usability heuristics for } \\
\text { testing gestural interaction }\end{array}$ & $\begin{array}{l}\text { Gesture learnability } \\
\text { Gesture cognitive Workload } \\
\text { Gesture adaptability } \\
\text { Gesture ergonomics }\end{array}$ & Sí & No & No & Sí & No \\
\hline
\end{tabular}




\begin{tabular}{|c|c|c|c|c|c|c|}
\hline $\begin{array}{l}\text { Assessing the reliability of heuristic } \\
\text { evaluation for website attractive- } \\
\text { ness and usability }\end{array}$ & $\begin{array}{l}\text { Heuristics for attractiveness and } \\
\text { aesthetic design } \\
\text { Content related heuristics }\end{array}$ & No & No & No & No & $\begin{array}{l}\text { Organización, } \\
\text { etiquetado y } \\
\text { navegación }\end{array}$ \\
\hline $\begin{array}{l}\text { Development of evaluation heuris- } \\
\text { tics for web service user experience }\end{array}$ & $\begin{array}{l}\text { H1: Usage and creation of composite services } \\
\text { H2: Cross-platform service access } \\
\text { H3: Social interaction and navigation } \\
\text { H4: Dynamic service features } \\
\text { H5: Context-aware services and contextually } \\
\text { enriched content } \\
\text { H6: General UX-related issues } \\
\text { H7: Findings outside heuristics 1-6 }\end{array}$ & Sí & Sí & No & Sí & Navegación \\
\hline $\begin{array}{l}\text { Establishing guidelines for user } \\
\text { quality of experience in ubiquitous } \\
\text { systems }\end{array}$ & $\begin{array}{l}\text { Make use of multimodal interaction } \\
\text { Designing continuous interaction in ubiqui- } \\
\text { tous services } \\
\text { Project to explore the characteristics of each } \\
\text { system } \\
\text { Know how to explore the characteristic of } \\
\text { invisibility } \\
\text { Design interfaces that minimize the effort of } \\
\text { attention } \\
\text { Designing for the user control } \\
\text { Designing interfaces for trust } \\
\text { Designing for error management } \\
\text { Design for accessibility }\end{array}$ & Sí & No & Sí & No & Navegación \\
\hline $\begin{array}{l}\text { Externalist philosophy and interac- } \\
\text { tion: proposal of heuristics for the } \\
\text { design of interactive systems }\end{array}$ & $\begin{array}{l}\text { 1. To propose interactive processes that work } \\
\text { simultaneously with several senses } \\
\text { 2. To provide embodied cognitive interaction } \\
\text { with the use of affordances } \\
\text { 3. To propose visual and metaphorical ele- } \\
\text { ments that are consistent with the users' men- } \\
\text { tal model of the system in order to facilitate } \\
\text { the cognitive process } \\
\text { 4. To provide answers to the user that are close } \\
\text { to their language and that have multimodal } \\
\text { characteristics } \\
\text { 5. To design devices and interfaces that can } \\
\text { be handled in a natural way, without the need } \\
\text { to deviate the user's attention from his main } \\
\text { activity at the time } \\
\text { 6. To design intuitive interactive interfaces } \\
\text { 7. To focus on the situation, regarding what it } \\
\text { takes to get the technology to work } \\
\text { 8. To reflect on the users' real needs while inte- } \\
\text { racting with the device or interface, in order to } \\
\text { provide only the desired interactions }\end{array}$ & Sí & No & No & No & Etiquetado \\
\hline $\begin{array}{l}\text { Towards the development of } \\
\text { usability heuristics for native smar- } \\
\text { tphone mobile applications }\end{array}$ & $\begin{array}{l}\text { Smart1: Provide immediate notification of } \\
\text { application status } \\
\text { Smart2: Use a theme and consistent terms, as } \\
\text { well as conventions and standards familiar to } \\
\text { the user } \\
\text { Smart3: Prevent errors where possible; Assist } \\
\text { users should an error occur } \\
\text { Smart4: Display an overlay pointing out the } \\
\text { main features when appropriate or requested } \\
\text { Smart5: Each interface should focus on one task } \\
\text { Smart6: Design a visually pleasing interface } \\
\text { Smart7: Intuitive interfaces make for easier } \\
\text { user journeys } \\
\text { Smart8: Design a clear navigable path to task } \\
\text { completion } \\
\text { Smart9: Allow configuration options and } \\
\text { shortcuts } \\
\text { Smart10: Cater for diverse mobile environments } \\
\text { Smart11: Facilitate easier input } \\
\text { Smart12: Use the camera, microphone and } \\
\text { sensors when appropriate to lessen the users' } \\
\text { workload } \\
\text { Smart13: Create an aesthetic and identifiable } \\
\text { icon }\end{array}$ & $\begin{array}{l}\text { Sí (nativo } \\
\text { móvil) }\end{array}$ & Sí & No & No & $\begin{array}{l}\text { Navegación y } \\
\text { etiquetado }\end{array}$ \\
\hline
\end{tabular}




\begin{tabular}{|c|c|c|c|c|c|c|}
\hline $\begin{array}{l}\text { Validation of navigation guide- } \\
\text { lines for improving usability in the } \\
\text { mobile web }\end{array}$ & $\begin{array}{l}\text { 1. Identification of links. Links should be easily } \\
\text { recognizable by the user in mobile devices } \\
\text { 2. Distinguishing adjacent links from each } \\
\text { other } \\
\text { 3. Distinguishing navigation links from trans- } \\
\text { actions } \\
\text { 4. Self-explanatory link cues } \\
\text { 5. Using familiar terminology for navigation } \\
\text { links } \\
\text { 6. Using descriptive link labels } \\
\text { 7. Highlighting previously visited links } \\
\text { 8. Marking links to special targets } \\
\text { 9. Marking links opening new windows } \\
\text { 10. Distinguishing navigation links from } \\
\text { controls } \\
\text { 11. Distinguishable within page links } \\
\text { 12. Link length } \\
\text { 13. Redundant links } \\
\text { 14. Avoiding link overload } \\
\text { 15. Page titles as bookmarks }\end{array}$ & $\begin{array}{l}\text { Sí (nativo } \\
\text { móvil) }\end{array}$ & Sí & No & Sí & $\begin{array}{c}\text { Navegación y } \\
\text { etiquetado }\end{array}$ \\
\hline
\end{tabular}

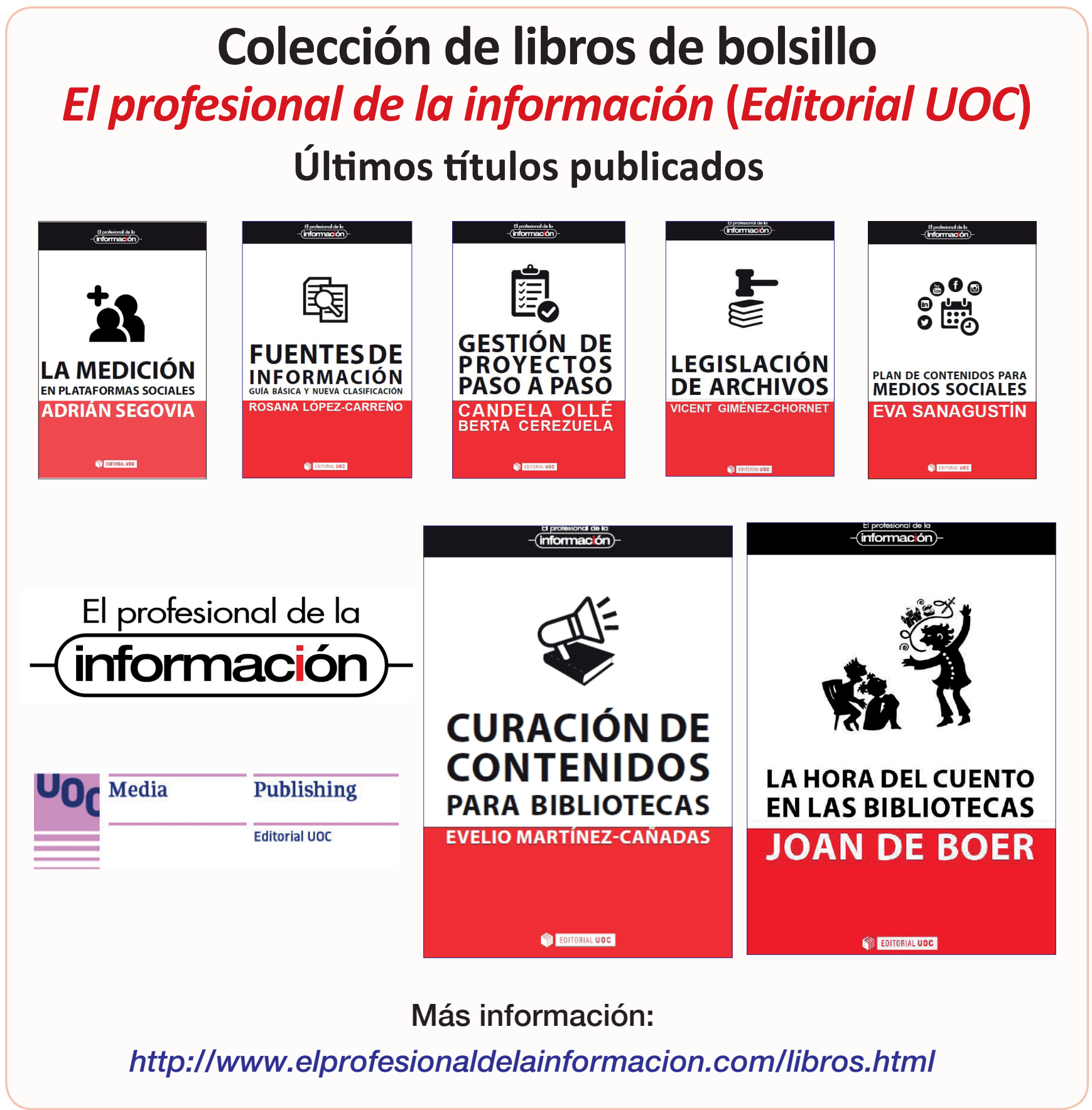

